\title{
Blistering in Bothrops atrox envenomings: Evidence of antivenom and inflammatory factors in the bite site
}

$\underline{\text { Sarah N. C. Gimenes }}{ }^{1}$; Jacqueline de A. G. Sachett ${ }^{2}$; Mônica Colombini ${ }^{1}$; Luciana A. Freitas-de-Sousa ${ }^{1}$; Hiochelson N. dos S. Ibiapina² ${ }^{2}$ Allyson G. da Costa ${ }^{2}$; Monique F. Santana ${ }^{2}$, Jeong-Jin Park ${ }^{4}$; Nicholas E. Sherman ${ }^{4}$, Luiz C. de L. Ferreira ${ }^{3}$, Fan H. Wen ${ }^{1}$; Wuelton M. Monteiro ${ }^{2}$; Ana M. Moura da Silva ${ }^{1}$; Jay W. Fox ${ }^{4}$

${ }^{1}$ Laboratório de Imunopatologia, Instituto Butantan, São Paulo, SP, Brazil

2 Escola Superior de Ciências da Saúde, Universidade do Estado do Amazonas, Manaus, AM, Brazil

${ }_{3}^{3}$ Departamento de Ensino e Pesquisa, Fundação de Dermatologia Alfredo da Matta, Manaus, AM, Brazil

${ }^{4}$ University of Virginia, Charlottesville, VA, USA

\section{Introduction}

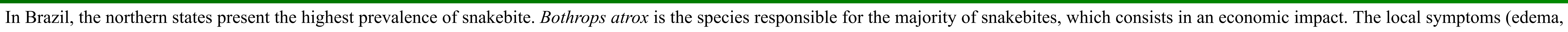

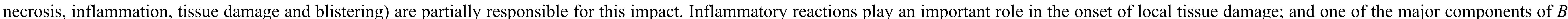

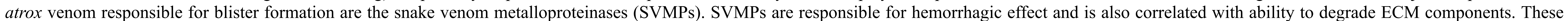

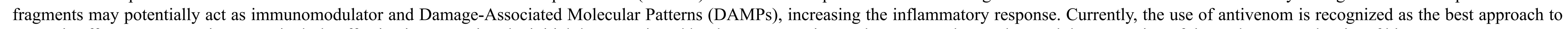
systemic effects. However, is not particularly effective in preventing the initial damage trigged by the venom toxins, and consequently we observed the worsening of tissue damage at the site of bite.

\section{Aims}

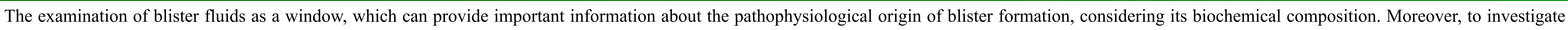
the possible reasons of limited protective efficiency of antivenom to be able to treat the local damage in $B$. atrox envenoming.

\section{Results}

Five patients were attended at Tropical Medicine Hospital, Manaus, Brazil (CAAE 53192516.8.0000.0005). They were classified according to the clinical data: moderate (patients 1, 2 and 3) and severe (patients 4 and 5) accident. The clinical data provide insights into the local symptoms experienced in this patient cohort. The presence of moderate to severe edema and elevated levels of LDH ( $>190 \mathrm{UI} / \mathrm{I}$ ), which is a marker indicative of tissue damage, was observed during the whole time of hospitalization (Table 1). The histological examination of tissue at the local site corroborated the clinical data, showing an acute inflammatory and hemorrhage in the area around the snakebite (Figure 1).
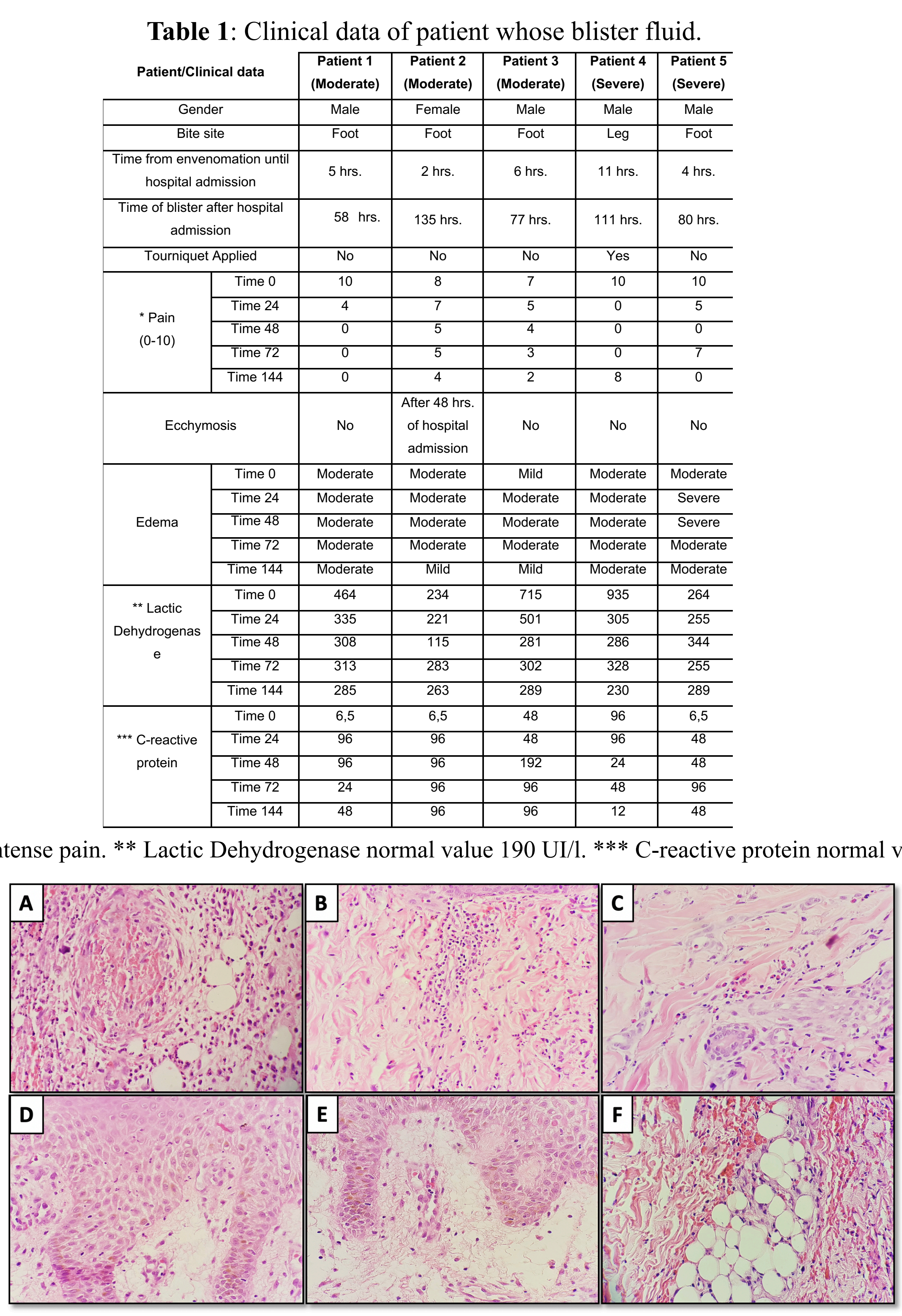

Figure 1: Illustrative figure of local damage. A, B and C) vascular thrombosis associated with vessel disruption and inflammatory infiltrate of mainly
eosinophils and neutrophils. D, E and F) exocytosis of neutrophils in the epidermal layer associated with the presence of edema and papillary edem and intense erythrocyte extravasation in the deep dermis layer, associated with mononuclear inflammatory infiltrate.

The proteomic data of the blister fluids correlated with previous blister fluid studies showing the presence of ECM and coagulation's proteins (Figure 2A and B), as well as DAMPs and immunomodulators (Figure 2C and D).
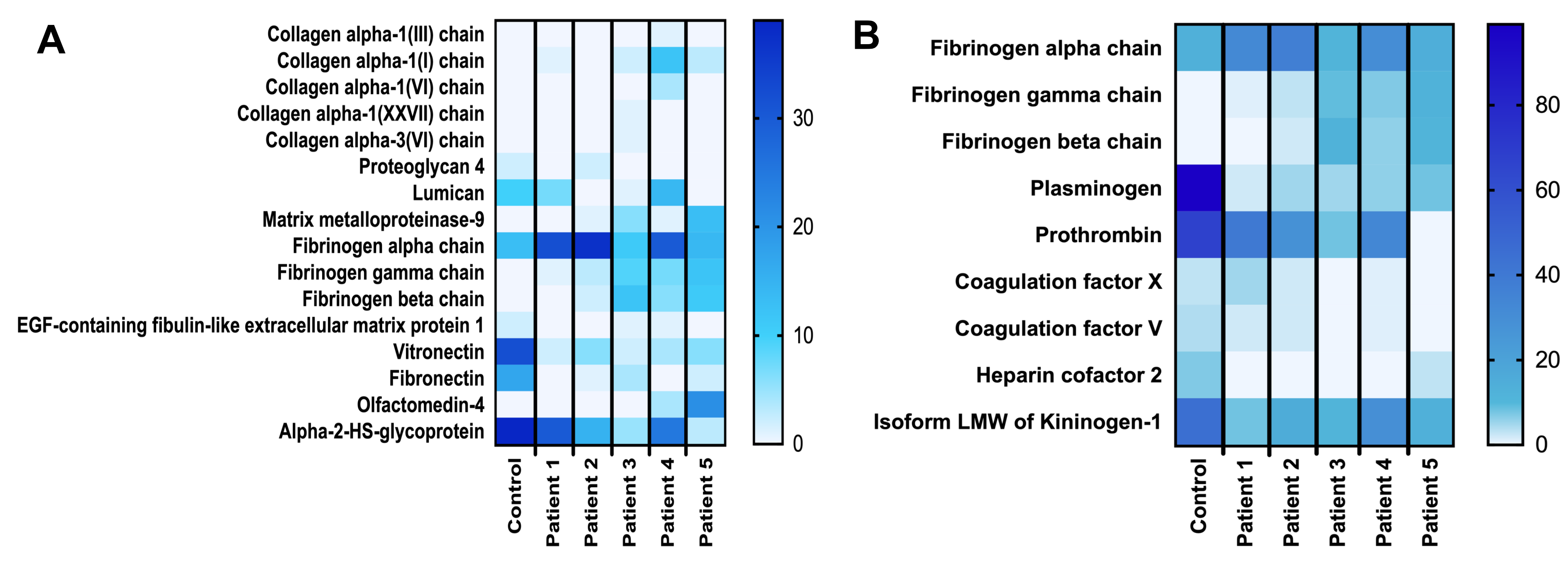

C
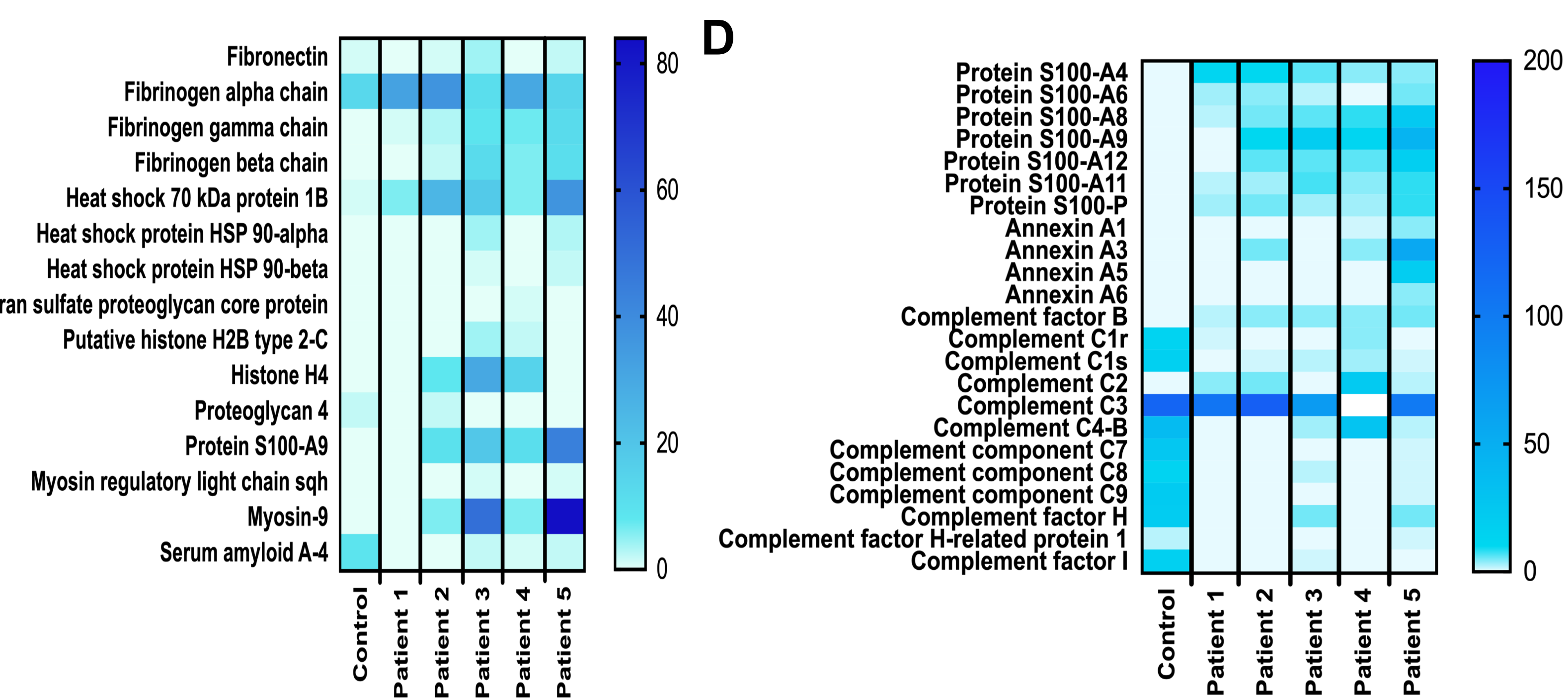

Figure 2: Proteomic data from blister fluids. A) Proteins present in blister fluid related to extracellular matrix; B) Proteins present in blister fluid related with coagulation; $\mathbf{C}$ ) DAMPs and D) Immunomodulators. Proteins were identified from the raw data using the Sequest search algorithm
Proteome Discovery against human basic database. The bar indicates the value of normalized total spectra count.
The blister composition was observed to be similar among the patients regardless of the clinical severity of envenomation. An unprecedented additional finding was that we identified venom (Figure 3A) and antivenom proteins (Figure 3B) in the bite site by ELISA. The venom was quantified in the fluid a significant time after envenommation (up to 135 hours), suggesting a slow clearance of venom at the site of bite, which might have influence on local tissue well after the time of envenomation (Figure 3A)
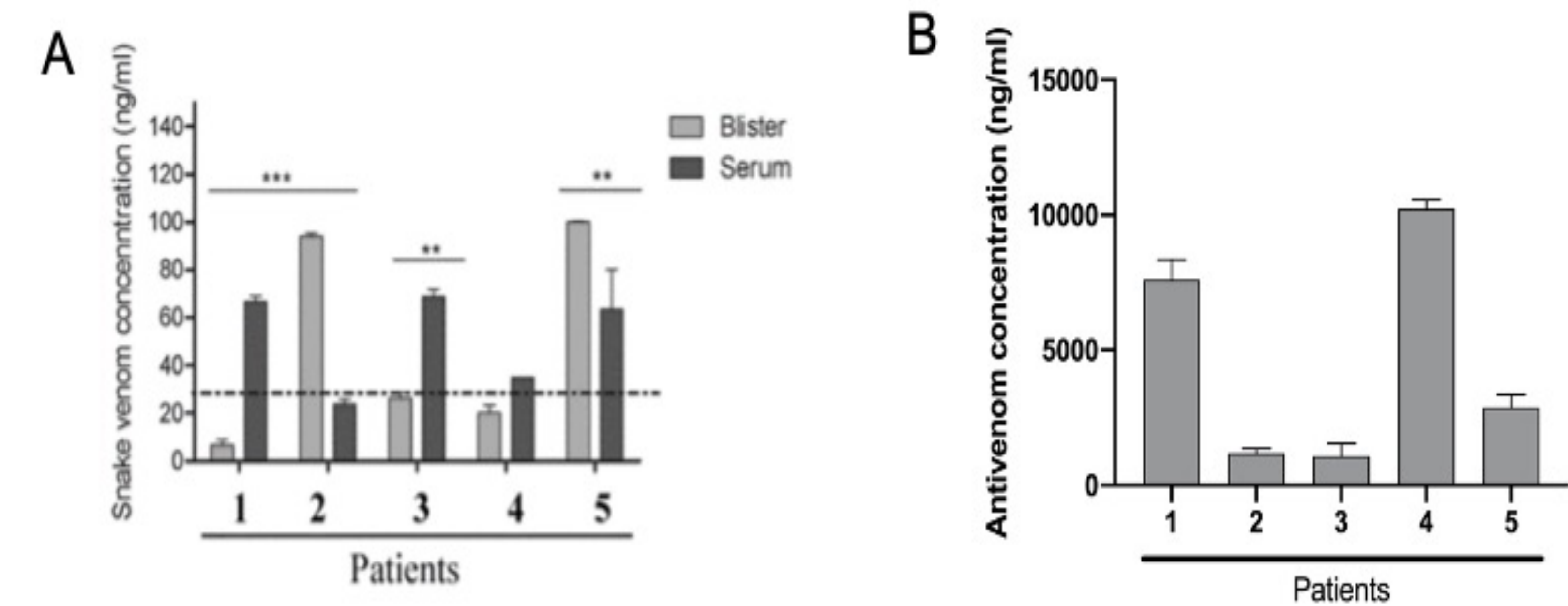

Figure 3: Quantitative analysis of snake venom and antivenom by ELISA. A) Quantitative analysis of antivenom presence in the Blister fluid an cerculating serum at the time of patient admission. B) Quantitative analysis of Bothrops atrox venom presence in the Blister fluid and circulating serum at the time of patient admission.

Antibodies from the administered antivenom identified in the blister fluids were shown capable of binding venom proteins by Western blotting (Figure 4). Thus, blister fluid antibodies should be capable of neutralizing the any venom components in the fluid. However, taken together, these findings suggest that although blistering is a delayed phenomenon of envenomation, its likely pathophysiological origins occur in advance of antivenom administration and venom neutralization at the site of envenomation and continues despite the eventual neutralization of venom.
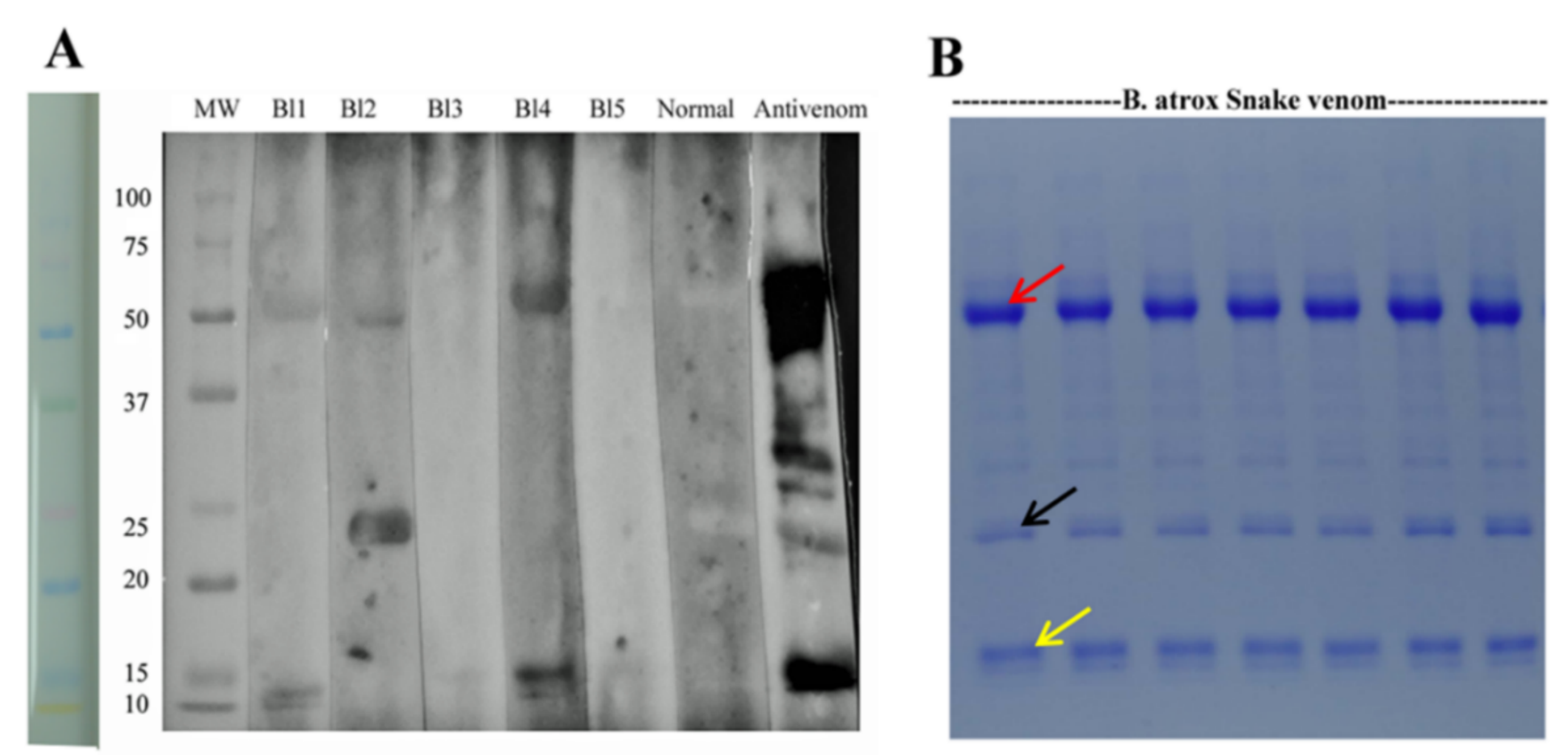

Figure 4: Analysis of the antivenom in Blister by Western Blotting. A) Western Blotting demonstrating the recognition of $B$. atrox venom proteins by the blister content. MW: Molecular Weight standard, BI1. Bhiter Patient 1; B12: Blister Patient 2; B13: Blister Patient 3; B14. Blister Patient 4; BI5; with $B$. atrox venom used for protein transferred in western blotting assay. Red arrow represents range of SVMP P-III; Black arrow represents range of SVMP P-I; Yellow arrow represents range of Phospholipases $\mathrm{A}_{2}$

\section{Conclusion}

We demonstrates that antivenom and venom reach and stay even after long time on the local lesions in B. atrox snakebite. However, the presence of antivenom in the blister did not prevent the severity of tissue damage or blister formation. It suggest that the blister is an independent process, and the pathophysiology of blister formation is more related to the presence of proinflammatory molecules released by the toxins action since the first moments after venom inoculation (Figure 4). Our studies underscore the need to develop rapid, in situ therapeutics to eliminate or at least attenuate the local effects of envenomation.

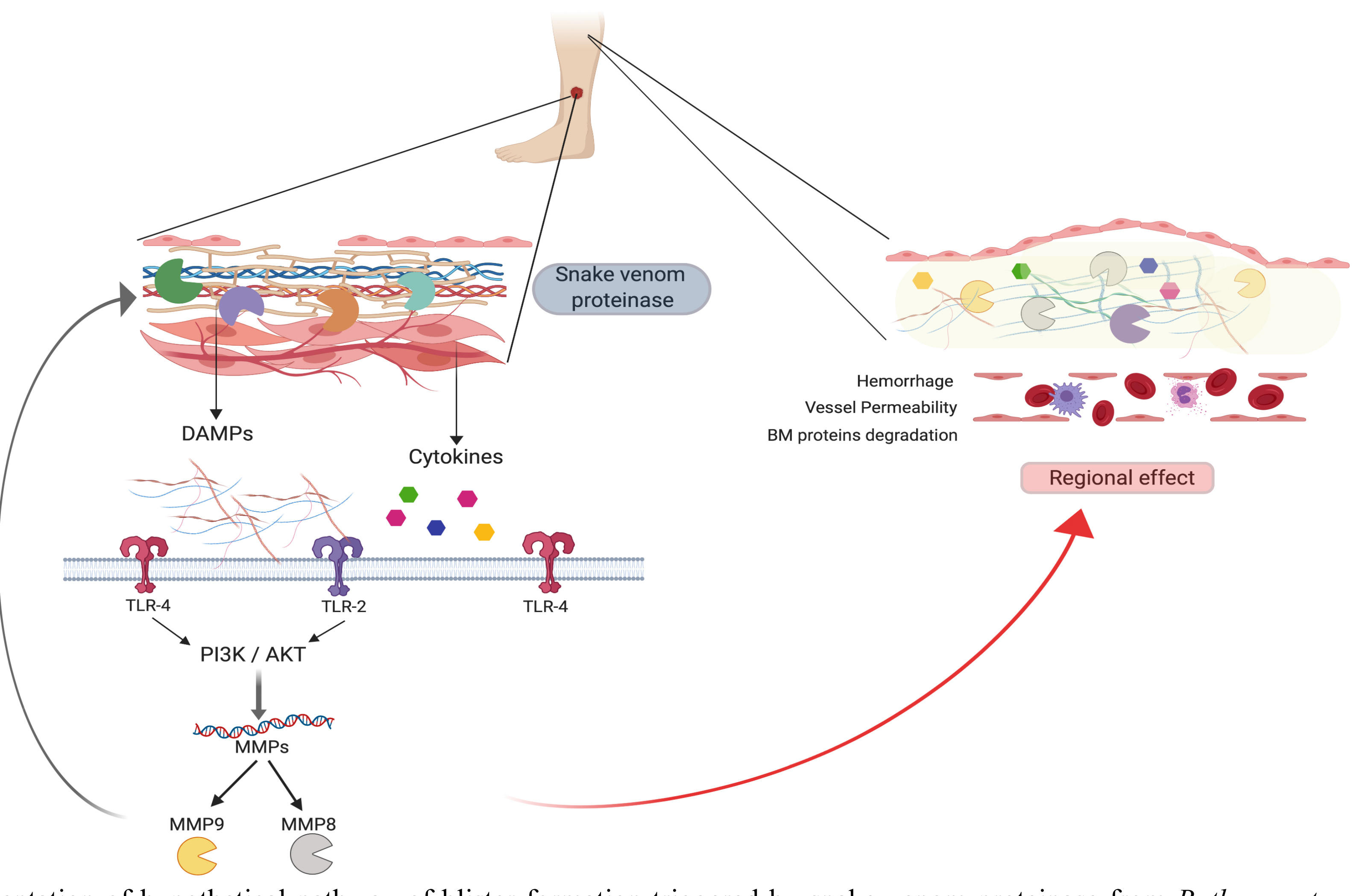

Figure 4: Representation of hypothetical pathway or
BioRender Standard Academic software was used. 